\title{
On the complete convergence for arrays of rowwise $\psi$-mixing random variables
}

\section{Yong-Feng $\mathrm{Wu}^{1}$ and Hui Ding ${ }^{2^{*}}$}

"Correspondence:
dinghuicz@126.com
${ }^{2}$ School of Mathematical Sciences,
Chuzhou University, Chuzhou,
239000, P.R. China
Full list of author information is
available at the end of the article

${ }^{*}$ Correspondence: dinghuicz@126.com

Full list of author information is

\begin{abstract}
Some sufficient conditions for complete convergence for maximal weighted sums $\max _{1 \leq j \leq n}\left|\sum_{k=1}^{j} a_{n k} X_{n k}\right|$ and weighted sums $\sum_{k=1}^{n} a_{n k} X_{n k}$ are presented, where $\left\{X_{n k}, 1 \leq k \leq n, n \geq 1\right\}$ is an array of rowwise $\psi$-mixing random variables, and $\left\{a_{n k}, 1 \leq k \leq n, n \geq 1\right\}$ is an array of constants. The obtained results extend and improve the corresponding result in the previous literature.
\end{abstract}

MSC: $60 \mathrm{~F} 15$

Keywords: $\psi$-mixing random variable; weighted sums; complete convergence

\section{Introduction}

The following notion was given firstly by Hsu and Robbins [1].

Definition 1.1 A sequence of random variables $\left\{U_{n}, n \geq 1\right\}$ is said to converge completely to a constant $\theta$ if for any $\varepsilon>0$,

$$
\sum_{n=1}^{\infty} P\left(\left|U_{n}-\theta\right|>\varepsilon\right)<\infty
$$

In this case, we write $U_{n} \rightarrow \theta$ completely. In view of the Borel-Cantelli lemma, the result above implies that $U_{n} \rightarrow \theta$ almost surely. Therefore, the complete convergence is a very important tool in establishing almost sure convergence of summation of random variables as well as weighted sums of random variables.

Let $\left\{X_{n}, n \geq 1\right\}$ be a sequence of random variables, defined on a probability space $(\Omega, \mathcal{F}, P)$, and denote $\sigma$-algebras

$$
\mathcal{F}_{n}^{m}=\sigma\left(X_{k}, n \leq k \leq m\right), \quad 1 \leq n \leq m \leq \infty
$$

As usual, for a $\sigma$-algebra $\mathcal{F}$, we denote by $\mathcal{L}^{2}(\mathcal{F})$ the class of all $\mathcal{F}$-measurable random variables with the finite second moment. Given $\sigma$-algebras $\mathcal{A}, \mathcal{B}$ in $\mathcal{F}$, let

$$
\begin{aligned}
& \psi(\mathcal{A}, \mathcal{B})=\sup _{A \in \mathcal{A}, B \in \mathcal{B}, P(A) P(B)>0}\left|\frac{P(A B)}{P(A) P(B)}-1\right|, \\
& \varphi(\mathcal{A}, \mathcal{B})=\sup _{A \in \mathcal{A}, B \in \mathcal{B}, P(A)>0}|P(B \mid A)-P(B)| .
\end{aligned}
$$

๑ 2013 Wu and Ding; licensee Springer. This is an Open Access article distributed under the terms of the Creative Commons Attribution License (http://creativecommons.org/licenses/by/2.0), which permits unrestricted use, distribution, and reproduction in any medium, provided the original work is properly cited. 
Define the mixing coefficients by

$$
\psi(n)=\sup _{k \geq 1} \psi\left(\mathcal{F}_{1}^{k}, \mathcal{F}_{k+n}^{\infty}\right), \quad \varphi(n)=\sup _{k \geq 1} \varphi\left(\mathcal{F}_{1}^{k}, \mathcal{F}_{k+n}^{\infty}\right), \quad n \geq 0 .
$$

The concepts of $\psi$-mixing and $\varphi$-mixing random variables were introduced by Blum et al. [2] and Dobrushin [3], respectively.

Definition 1.2 A sequence of random variables $\left\{X_{n}, n \geq 1\right\}$ is said to be a $\psi$-mixing ( $\varphi$-mixing) sequence of random variables if $\psi(n) \downarrow 0(\varphi(n) \downarrow 0)$ as $n \rightarrow \infty$.

Clearly, from the definition above, we know that the independence implies $\psi$-mixture and $\varphi$-mixture. It is easily seen that the $\psi$-mixing condition is stronger than the $\varphi$-mixing. Therefore, the family of $\psi$-mixing is a special case of $\varphi$-mixing. Years after the appearance of Dobrushin [3], many works of investigation concerning the convergence properties of $\varphi$-mixing random variables have emerged. We refer the reader to Ibragimov [4], Cogburn [5], Sen [6], Choi and Sung [7], Utev [8], Chen [9], Shao [10], Rüdiger [11], Chen et al. [12], Zhou [13], Wang et al. [14, 15], Guo [16].

However, according to our knowledge, few papers discuss the subjects for sequences or arrays of $\psi$-mixing random variables except Blum et al. [2], Bradley [17], Yang [18], Wu and Zhu [19], Wang et al. [14, 15], and Yang and Liu [20]. The goal of this paper is to study a complete convergence for arrays of rowwise $\psi$-mixing random variables.

Then we recall that the following concept of stochastic domination is a slight generalization of identical distribution.

Definition 1.3 An array of rowwise random variables $\left\{X_{n k}, 1 \leq k \leq n, n \geq 1\right\}$ is said to be stochastically dominated by a nonnegative random variable $X$ (write $\left\{X_{n k}\right\} \prec X$ ) if there exists a constant $C>0$ such that

$$
\sup _{n, k} P\left(\left|X_{n k}\right|>x\right) \leq C P(X>x), \quad \forall x>0 .
$$

Stochastic dominance of $\left\{X_{n k}, 1 \leq k \leq n, n \geq 1\right\}$ by the random variable $X$ implies that $E\left|X_{n k}\right|^{p} \leq C E X^{p}$ if the $p$-moment of $X$ exists, i.e., if $E X^{p}<\infty$.

Hu et al. [21] obtained the following result in the complete convergence.

Theorem $\mathbf{A}$ Let $\left\{X_{n k}, 1 \leq k \leq n, n \geq 1\right\}$ be an array of rowwise independent random variables with $E X_{n k}=0$. Suppose that $\left\{X_{n k}, 1 \leq k \leq n, n \geq 1\right\}$ are uniformly bounded by some random variable $X$. If $E|X|^{2 p}<\infty$ for some $1 \leq p<2$, then

$$
n^{-1 / p} \sum_{k=1}^{n} X_{n k} \rightarrow 0 \quad \text { completely. }
$$

Taylor et al. [22], Baek et al. [23] extended and generalized Theorem A to rowwise negatively dependent $(\mathrm{ND})$ random variables.

The main purpose of this article is to discuss the complete convergence for weighted sums of $\psi$-mixing random variables. We shall extend Theorem A by considering $\psi$-mixing instead of independent. It is worthy to point out that our main methods differ from those used by Hu et al. [21]. 
Below, $C$ will be used to denote various positive constants, whose value may vary from one application to another. For a finite set $A$, the symbol $\sharp(A)$ denotes the number of elements in the set $A . I_{(A)}$ will indicate the indicator function of $A$.

\section{Main results and some lemmas}

Now, we state our main results. The proofs will be given in Section 3.

Theorem 2.1 Let $\left\{X_{n k}, 1 \leq k \leq n, n \geq 1\right\}$ be an array of rowwise $\psi$-mixing random variables with $\sum_{m=1}^{\infty} \psi(m)<\infty$ and $E X_{n k}=0$. Suppose that $\left\{X_{n k}\right\} \prec X$ and $E X^{2 p}<\infty$ for some $p>0$. Let $\left\{a_{n k}, 1 \leq k \leq n, n \geq 1\right\}$ be a real numbers array satisfying $\max _{1 \leq k \leq n}\left|a_{n k}\right|=O\left(n^{-\alpha}\right)$ for some $\alpha>1 /(2 p)$. Furthermore, when $p \geq 1$, we suppose that there exists a constant $\theta>0$ such that $\sum_{k=1}^{n} a_{n k}^{2} \leq \mathrm{Cn}^{-\theta}$. Then

$$
\sum_{n=1}^{\infty} n^{2 \alpha p-2} P\left(\max _{1 \leq j \leq n}\left|\sum_{k=1}^{j} a_{n k} X_{n k}\right|>\varepsilon\right)<\infty, \quad \forall \varepsilon>0 .
$$

Take $a_{n k}=n^{-1 / p}$ and $1 \leq p<2$ in Theorem 2.1, we can have the following corollary.

Corollary 2.1 Let $\left\{X_{n k}, 1 \leq k \leq n, n \geq 1\right\}$ be an array of rowwise $\psi$-mixing random variables with $\sum_{m=1}^{\infty} \psi(m)<\infty$ and $E X_{n k}=0$. Suppose that $\left\{X_{n k}\right\} \prec X$ and $E X^{2 p}<\infty$ for some $1 \leq p<2$, then

$$
\sum_{n=1}^{\infty} P\left(\max _{1 \leq j \leq n}\left|\sum_{k=1}^{j} X_{n k}\right|>n^{1 / p} \varepsilon\right)<\infty, \quad \forall \varepsilon>0 .
$$

Remark 2.1 Since the independence implies $\psi$-mixture, Theorem 2.1 and Corollary 2.1 hold for arrays of rowwise independent random variables. Therefore, Theorem 2.1 and Corollary 2.1 extend and improve Theorem A.

Theorem 2.2 Let $\left\{X_{n k}, 1 \leq k \leq n, n \geq 1\right\}$ be an array of rowwise $\psi$-mixing random variables with $E X_{n k}=0$. Suppose that $\left\{X_{n k}\right\} \prec X$ and $E X^{2 p}<\infty$ for some $p \geq 1$. Let $\left\{a_{n k}, 1 \leq\right.$ $k \leq n, n \geq 1$ be a real numbers array satisfying $\max _{1 \leq k \leq n}\left|a_{n k}\right|=O\left(n^{-\alpha}\right)$ for some $\alpha>1 /(2 p)$. Suppose that the following statements hold.

(i) There exists a positive constant $\lambda<\min \left\{\frac{1}{2 p}, \frac{2 \alpha p-1}{4 p}\right\}$ such that $\sum_{n=1}^{\infty} \psi^{\frac{\lambda}{1-\lambda}}(n)<\infty$;

(ii) $\log n \sum_{k=1}^{n} a_{n k}^{2}=o$ (1) if $\frac{1}{2 p}<\alpha \leq \frac{1}{2}$. Then

$$
\sum_{n=1}^{\infty} n^{2 \alpha p-2} P\left(\left|\sum_{k=1}^{n} a_{n k} X_{n k}\right|>\varepsilon\right)<\infty, \quad \forall \varepsilon>0 .
$$

Remark 2.2 Compared with Theorem 2.1, Theorem 2.2 requires a stronger mixing rate, but weakens the requirement of $\sum_{k=1}^{n} a_{n k}^{2}$. In fact, $\log n \sum_{k=1}^{n} a_{n k}^{2}=o(1)$ holds if $\sum_{k=1}^{n} a_{n k}^{2} \leq$ $\mathrm{Cn}^{-\theta}, \theta>0$.

Now, we state some lemmas which will be used in the proofs of our main results.

Lemma 2.1 (Wang et al. [14,15]) Let $\left\{X_{n}, n \geq 1\right\}$ be a sequence of $\psi$-mixing random variables satisfying $\sum_{m=1}^{\infty} \psi(m)<\infty, q \geq 2$. Assume that $E X_{n}=0$ and $E\left|X_{n}\right|^{q}<\infty$ for each 
$n \geq 1$. Then there exists a constant $C$ depending only on $q$ and $\psi(\cdot)$ such that

$$
E \max _{1 \leq j \leq n}\left|\sum_{k=1}^{j} X_{k}\right|^{q} \leq C\left\{\sum_{k=1}^{n} E\left|X_{k}\right|^{q}+\left(\sum_{k=1}^{n} E X_{k}^{2}\right)^{q / 2}\right\} .
$$

Lemma 2.2 (Yang [18]) Let $\left\{X_{n}, n \geq 1\right\}$ be a sequence of $\psi$-mixing random variables with $E X_{k}=0,\left|X_{k}\right| \leq d<\infty$ a.s., $k=1,2, \ldots, 0<\lambda<1, m=\left[n^{\lambda}\right]$. Then $\forall \varepsilon>0$,

$$
P\left(\left|\sum_{k=1}^{n} X_{k}\right|>\varepsilon\right) \leq 2 e C_{1} \exp \left\{-t \varepsilon+C_{2} t^{2} B_{n}\right\},
$$

where $B_{n}=\sum_{k=1}^{n} E X_{k}^{2}, t m d \leq 1 / 4, C_{1}=\exp \left\{2 e n^{1-\lambda} \psi(m)\right\}, C_{2}=4\left(1+4 \sum_{k=1}^{2 m} \psi(k)\right)$.

Lemma 2.3 Let $\left\{X_{n}, n \geq 1\right\}$ be a sequence of $\psi$-mixing random variables, and let $A_{j}=$ $\left\{\left|X_{j}\right| \geq x_{j}\right\}, x_{j} \in \mathbb{R}^{+}, j=1,2, \ldots, N$, then

$$
P\left(A_{1}, A_{2}, \ldots, A_{N}\right) \leq(1+\psi(1))^{N} \prod_{j=1}^{N} P\left(A_{j}\right)
$$

Proof By the definition of $\psi$-mixing, we have

$$
\begin{aligned}
P\left(A_{1}, A_{2}, \ldots, A_{N}\right) & \leq(1+\psi(1)) P\left(A_{1}\right) P\left(A_{2}, \ldots, A_{N}\right) \\
& \leq \cdots \\
& \leq(1+\psi(1))^{N} P\left(A_{1}\right) P\left(A_{2}\right) \cdots P\left(A_{N}\right) .
\end{aligned}
$$

The proof is complete.

\section{Proofs}

In this section, we state the proofs of our main results.

Proof of Theorem 2.1 Let $S_{n j}=\sum_{k=1}^{j} a_{n k} X_{n k}, 1 \leq j \leq n$. Since $a_{n k}=a_{n k}^{+}-a_{n k}^{-}$, without loss of generality, we may assume that $0<a_{n k} \leq C n^{-\alpha}$. Let $0<\rho<\frac{(2 \alpha p-1)(N-1)}{2 p N}$, where $N$ is a positive integer with $N>1$. Let

$$
\begin{aligned}
& X_{n k}^{\prime}=X_{n k} I_{\left(a_{n k}\left|X_{n k}\right| \leq n^{-\rho}\right)}, \quad X_{n k}^{\prime \prime}=X_{n k} I_{\left(a_{n k}\left|X_{n k}\right|>\varepsilon / N\right)}, \\
& X_{n k}^{\prime \prime \prime}=X_{k}-X_{n k}^{\prime}-X_{n k}^{\prime \prime}=X_{n k} I_{\left(n^{-} \rho_{<a_{n k} \mid}\left|X_{n k}\right| \leq \varepsilon / N\right)}, \\
& S_{n j}^{\prime}=\sum_{k=1}^{j} a_{n k} X_{n k}^{\prime}, \quad S_{n j}^{\prime \prime}=\sum_{k=1}^{j} a_{n k} X_{n k}^{\prime \prime}, \quad S_{n j}^{\prime \prime \prime}=\sum_{k=1}^{j} a_{n k} X_{n k}^{\prime \prime \prime} .
\end{aligned}
$$

Firstly, we prove $\sum_{n=1}^{\infty} n^{2 \alpha p-2} P\left(\max _{1 \leq j \leq n}\left|S_{n j}^{\prime}\right|>\varepsilon\right)<\infty$. By $\left\{X_{n}\right\} \prec X$, we know that $E\left|X_{n k}\right|^{2 p} \leq E X^{2 p}<\infty$. If $0<p \leq 1 / 2$, we have

$$
\begin{aligned}
\max _{1 \leq j \leq n}\left|\sum_{k=1}^{j} a_{n k} E X_{n k}^{\prime}\right| & \leq \sum_{k=1}^{n} a_{n k}\left|E X_{n k}^{\prime}\right| \leq \sum_{k=1}^{n} a_{n k} E\left|X_{n k}\right| I_{\left(a_{n k}\left|X_{n k}\right| \leq n^{-\rho}\right)} \\
& \leq \sum_{k=1}^{n} \frac{a_{n k}^{2 p} E\left|X_{n k}\right|^{2 p}}{n^{-2 \rho p}} n^{-\rho} \leq C n^{\rho(2 p-1)-(2 \alpha p-1)} \rightarrow 0, \quad n \rightarrow \infty .
\end{aligned}
$$


If $p>1 / 2$, by $E X_{n k}=0$ and $\rho<\frac{2 \alpha p-1}{2 p}<\frac{2 \alpha p-1}{2 p-1}$, we also have

$$
\begin{aligned}
\max _{1 \leq j \leq n}\left|\sum_{k=1}^{j} a_{n k} E X_{n k}^{\prime}\right| & \leq \sum_{k=1}^{n} a_{n k}\left|E X_{n k}^{\prime}\right| \leq \sum_{k=1}^{n} a_{n k} E\left|X_{n k}\right| I_{\left(a_{n k}\left|X_{n k}\right|>n^{-\rho}\right)} \\
& \leq \sum_{k=1}^{n} \frac{a_{n k}^{2 p} E\left|X_{n k}\right|^{2 p}}{n^{-2 \rho p}} n^{-\rho} \leq C n^{\rho(2 p-1)-(2 \alpha p-1)} \rightarrow 0, \quad n \rightarrow \infty .
\end{aligned}
$$

Therefore, we know that (1) holds for $p>0$. Let $S_{n j}^{*}=\sum_{k=1}^{j} a_{n k}\left(X_{n k}^{\prime}-E X_{n k}^{\prime}\right)$. To prove $\sum_{n=1}^{\infty} n^{2 \alpha p-2} P\left(\max _{1 \leq j \leq n}\left|S_{n j}^{\prime}\right|>\varepsilon\right)<\infty$, it suffices to show that $\sum_{n=1}^{\infty} n^{2 \alpha p-2} P\left(\max _{1 \leq j \leq n}\left|S_{n j}^{*}\right|>\right.$ $\varepsilon)<\infty$.

If $0<p<1$, by Markov's inequality and Lemma 2.1, we have

$$
\begin{aligned}
& \sum_{n=1}^{\infty} n^{2 \alpha p-2} P\left(\max _{1 \leq j \leq n}\left|S_{n j}^{*}\right|>\varepsilon\right) \\
& \quad \leq C \sum_{n=1}^{\infty} n^{2 \alpha p-2} E\left(\max _{1 \leq j \leq n}\left|S_{n j}^{*}\right|\right)^{2} \leq C \sum_{n=1}^{\infty} n^{2 \alpha p-2} \sum_{k=1}^{n} a_{n k}^{2} E\left(X_{n k}^{\prime}\right)^{2} \\
& \quad \leq \sum_{n=1}^{\infty} n^{2 \alpha p-2} \sum_{k=1}^{n} a_{n k}^{2 p} E\left(\left|a_{n k} X_{n k}^{\prime}\right|^{2-2 p}\left|X_{n k}^{\prime}\right|^{2 p}\right) \\
& \quad \leq \sum_{n=1}^{\infty} n^{2 \alpha p-2} n^{-\rho(2-2 p)} \sum_{k=1}^{n} a_{n k}^{2 p} E\left|X_{n k}^{\prime}\right|^{2 p} \leq C \sum_{n=1}^{\infty} n^{-1-2 \rho(1-p)}<\infty .
\end{aligned}
$$

If $p \geq 1$, take $q>\max \{2 p, 2(2 \alpha p-1) / \theta\}$. By $q>2$ and Lemma 2.1, we have

$$
\begin{aligned}
& \sum_{n=1}^{\infty} n^{2 \alpha p-2} P\left(\max _{1 \leq j \leq n}\left|S_{n j}^{*}\right|>\varepsilon\right) \\
& \leq \sum_{n=1}^{\infty} n^{2 \alpha p-2}\left[\sum_{k=1}^{n} a_{n k}^{q} E\left|X_{n k}^{\prime}\right|^{q}+\left(\sum_{k=1}^{n} a_{n k}^{2} E\left(X_{n k}^{\prime}\right)^{2}\right)^{q / 2}\right] .
\end{aligned}
$$

By a similar argument as in the proof of (2) (replacing exponent 2 into $q$ ), we can get

$$
\sum_{n=1}^{\infty} n^{2 \alpha p-2} \sum_{k=1}^{n} a_{n k}^{q} E\left|X_{n k}^{\prime}\right|^{q}<\infty .
$$

Note that $E\left|X_{n k}^{\prime}\right|^{2} \leq E X^{2}<\infty$ and the definition of $q$, we have

$$
\begin{aligned}
& \sum_{n=1}^{\infty} n^{2 \alpha p-2}\left(\sum_{k=1}^{n} a_{n k}^{2} E\left(X_{n k}^{\prime}\right)^{2}\right)^{q / 2} \\
& \leq \sum_{n=1}^{\infty} n^{2 \alpha p-2}\left(\sum_{k=1}^{n} a_{n k}^{2}\right)^{q / 2}\left(E X^{2}\right)^{q / 2} \leq C \sum_{n=1}^{\infty} n^{-1+(2 \alpha p-1)-\theta q / 2}\left(E X^{2}\right)^{q / 2}<\infty .
\end{aligned}
$$


From (3)-(5), we know that (2) still holds for $p \geq 1$. By (1) and (2), we have

$$
\sum_{n=1}^{\infty} n^{2 \alpha p-2} P\left(\max _{1 \leq j \leq n}\left|S_{n j}^{\prime}\right|>\varepsilon\right)<\infty
$$

Secondly, we prove $\sum_{n=1}^{\infty} n^{2 \alpha p-2} P\left(\max _{1 \leq j \leq n}\left|S_{n j}^{\prime \prime}\right|>\varepsilon\right)<\infty$. Let $\varphi_{n}(j)=\sharp\left\{1 \leq k \leq n: a_{n k}>\right.$ $\varepsilon /(j N)\}$ and $\phi_{j}=\left[(j C N / \varepsilon)^{1 / \alpha}\right]$, then

$$
\begin{aligned}
P\left(\max _{1 \leq j \leq n}\left|S_{n j}^{\prime \prime}\right|>\varepsilon\right) & \leq P\left(\bigcup_{k=1}^{n}\left\{a_{n k}\left|X_{n k}\right|>\varepsilon / N\right\}\right) \\
& \leq \sum_{k=1}^{n} P\left(a_{n k}\left|X_{n k}\right|>\varepsilon / N\right) \leq C \sum_{k=1}^{n} P\left(a_{n k} X>\varepsilon / N\right) \\
& =C \sum_{j=1}^{\infty} \sum_{k=1}^{n} P\left(a_{n k} X>\varepsilon / N, j-1 \leq X<j\right) \\
& \leq C \sum_{j=1}^{\infty} \varphi_{n}(j) P(j-1 \leq X<j) .
\end{aligned}
$$

By $a_{n k} X>\varepsilon / N$, we know $a_{n k}>\varepsilon /(j N)$. Note $a_{n k} \leq C n^{-\alpha}$, we have $n<(j C N / \varepsilon)^{1 / \alpha}$. Hence, we have $n \leq \phi_{j}$, then

$$
\begin{aligned}
\sum_{n=1}^{\infty} n^{2 \alpha p-2} P\left(\max _{1 \leq j \leq n}\left|S_{n j}^{\prime \prime}\right|>\varepsilon\right) & \leq C \sum_{n=1}^{\infty} n^{2 \alpha p-2} \sum_{j=1}^{\infty} \varphi_{n}(j) P(j-1 \leq X<j) \\
& \leq C \sum_{j=1}^{\infty} \sum_{n=1}^{\phi_{j}} n^{2 \alpha p-2} \varphi_{n}(j) P(j-1 \leq X<j) .
\end{aligned}
$$

Take $v \in\left(0, \frac{2 \alpha p-1}{\alpha}\right)$, then $\sum_{k=1}^{n} a_{n k}^{v} \geq \varphi_{n}(j) \varepsilon^{v} /(j N)^{\nu}$. From $\sum_{k=1}^{n} a_{n k}^{v} \leq C n^{1-\alpha \nu}$, we have

$$
\varphi_{n}(j) \leq N^{v} j^{v} / \varepsilon^{v} \sum_{k=1}^{n} a_{n k}^{v} \leq C n^{1-\alpha v} j^{v}
$$

Therefore, by (7) and (8), we have

$$
\sum_{n=1}^{\infty} n^{2 \alpha p-2} P\left(\max _{1 \leq j \leq n}\left|S_{n j}^{\prime \prime}\right|>\varepsilon\right) \leq C \sum_{j=1}^{\infty} \sum_{n=1}^{\phi_{j}} n^{2 \alpha p-\alpha \nu-1} j^{\nu} P(j-1 \leq X<j) .
$$

By the definition of $v$, we have $2 \alpha p-\alpha \nu-1>0$, then

$$
\sum_{n=1}^{\phi_{j}} n^{2 \alpha p-\alpha v-1} \leq \phi_{j}^{\alpha(2 p-v)} \leq C j^{2 p-v} .
$$

From (9), (10) and $E X^{2 p}<\infty$, then

$$
\sum_{n=1}^{\infty} n^{2 \alpha p-2} P\left(\max _{1 \leq j \leq n}\left|S_{n j}^{\prime \prime}\right|>\varepsilon\right) \leq C \sum_{j=1}^{\infty} j^{2 p} P(j-1 \leq X<j)<\infty .
$$


Finally, we prove $\sum_{n=1}^{\infty} n^{2 \alpha p-2} P\left(\max _{1 \leq j \leq n}\left|S_{n j}^{\prime \prime \prime}\right|>\varepsilon\right)<\infty$. Obviously, we know that $P\left(\max _{1 \leq j \leq n}\left|S_{n j}^{\prime \prime \prime}\right|>\varepsilon\right) \leq P\left(\sum_{k=1}^{n} a_{n k}\left|X_{n k}^{\prime \prime \prime}\right|>\varepsilon\right)$. Let $M=\sharp\left\{1 \leq k \leq n: n^{-\rho}<a_{n k}\left|X_{n k}\right| \leq \varepsilon / N\right\}$. We must let $M$ be at least $N$ such that $\sum_{k=1}^{n} a_{n k}\left|X_{n k}^{\prime \prime \prime}\right|>\varepsilon$. Take $W_{k}=\left\{a_{n k}\left|X_{n k}\right|>n^{-\rho}\right\}$, we have

$$
\begin{aligned}
P\left(\max _{1 \leq j \leq n}\left|S_{n j}^{\prime \prime \prime}\right|>\varepsilon\right) & \leq P\left(M \text { is at least } N \text {, such that } a_{n k}\left|X_{n k}\right|>n^{-\rho}\right) \\
& \leq \sum_{1 \leq i_{1}<i_{2}<\cdots<i_{N} \leq n} P\left(W_{i_{1}} W_{i_{2}} \cdots W_{i_{N}}\right) .
\end{aligned}
$$

By Lemma 2.3, we have

$$
P\left(W_{i_{1}} W_{i_{2}} \cdots W_{i_{N}}\right) \leq(1+\psi(1))^{N} P\left(W_{i_{1}}\right) P\left(W_{i_{2}}\right) \cdots P\left(W_{i_{N}}\right) .
$$

From (11) and (12), we have

$$
\begin{aligned}
& n^{2 \alpha p-2} P\left(\max _{1 \leq j \leq n}\left|S_{n j}^{\prime \prime \prime}\right|>\varepsilon\right) \\
& \leq(1+\psi(1))^{N} n^{2 \alpha p-2} \sum_{1 \leq i_{1}<i_{2}<\cdots<i_{N} \leq n} P\left(W_{i_{1}}\right) P\left(W_{i_{2}}\right) \cdots P\left(W_{i_{N}}\right) \\
& \quad \leq C n^{2 \alpha p-2}\left(\begin{array}{c}
n \\
N
\end{array}\right) P^{N}\left(X>a_{n k}^{-1} n^{-\rho}\right) \\
& \quad \leq C n^{2 \alpha p-2} n^{N} P^{N}\left(X>C^{-1} n^{-\rho+\alpha}\right) \\
& \leq C n^{-1-(2 \alpha p-1)(N-1)+2 \rho p N}\left(E X^{2 p}\right)^{N} .
\end{aligned}
$$

Noting that $0<\rho<\frac{(2 \alpha p-1)(N-1)}{2 p N}$. We have

$$
-(2 \alpha p-1)(N-1)+2 \rho p N<0,
$$

then

$$
\sum_{n=1}^{\infty} n^{2 \alpha p-2} P\left(\max _{1 \leq j \leq n}\left|S_{n j}^{\prime \prime \prime}\right|>\varepsilon\right) \leq C \sum_{n=1}^{\infty} n^{-1-(2 \alpha p-1)(N-1)+2 \rho p N}<\infty .
$$

The proof is completed.

Proof of Theorem 2.2 Following the notations of $X_{n k}^{\prime}, X_{n k}^{\prime \prime}$ and $X_{n k}^{\prime \prime \prime}$, but let

$$
\begin{array}{ll}
T_{n}^{\prime}=\sum_{k=1}^{n} a_{n k} X_{n k}^{\prime}, & T_{n}^{\prime \prime}=\sum_{k=1}^{n} a_{n k} X_{n k}^{\prime \prime}, \\
T_{n}^{\prime \prime \prime}=\sum_{k=1}^{n} a_{n k} X_{n k}^{\prime \prime \prime}, & T_{n}^{*}=\sum_{k=1}^{n} a_{n k}\left(X_{n k}^{\prime}-E X_{n k}^{\prime}\right) .
\end{array}
$$

Obviously, by following the methods used in the proof of (1), we have

$$
\left|\sum_{k=1}^{n} a_{n k} E X_{n k}^{\prime}\right| \rightarrow 0, \quad n \rightarrow \infty
$$


By similar arguments as in the proofs of

$$
\sum_{n=1}^{\infty} n^{2 \alpha p-2} P\left(\max _{1 \leq j \leq n}\left|S_{n j}^{\prime \prime}\right|>\varepsilon\right)<\infty \quad \text { and } \quad \sum_{n=1}^{\infty} n^{2 \alpha p-2} P\left(\max _{1 \leq j \leq n}\left|S_{n j}^{\prime \prime \prime}\right|>\varepsilon\right)<\infty
$$

we can prove

$$
\sum_{n=1}^{\infty} n^{2 \alpha p-2} P\left(\left|T_{n}^{\prime \prime}\right|>\varepsilon\right)<\infty \quad \text { and } \quad \sum_{n=1}^{\infty} n^{2 \alpha p-2} P\left(\left|T_{n}^{\prime \prime \prime}\right|>\varepsilon\right)<\infty
$$

Here, we omit the details. Therefore, we need only to show

$$
\sum_{n=1}^{\infty} n^{2 \alpha p-2} P\left(\left|T_{n}^{*}\right|>\varepsilon\right)<\infty
$$

Take $\lambda<\rho<\frac{(2 \alpha p-1)(N-1)}{2 p N}$, by $0<\lambda<\frac{1}{2 p}$ and $p \geq 1$, we know $0<\frac{\lambda}{1-\lambda}<1$. Hence, from condition (i), we have

$$
C_{2}=4\left(1+4 \sum_{k=1}^{2 m} \psi(k)\right) \leq 4\left(1+4 \sum_{k=1}^{\infty} \psi(k)\right)<\infty, \quad \psi(m)=o\left(m^{\frac{\lambda-1}{\lambda}}\right),
$$

where $m=\left[n^{\lambda}\right]$. Therefore, $C_{1}=\exp \left\{2 e n^{1-\lambda} \psi(m)\right\} \ll \exp \{2 e\}<\infty$.

Take $t=\frac{2 \alpha p \log n}{\varepsilon}$. Clearly, $t \leq n^{\rho-\lambda} / 8$ when $n$ is sufficiently large. Note that $\mid a_{n k}\left(X_{n k}^{\prime}-\right.$ $\left.E X_{n k}^{\prime}\right) \mid \leq 2 n^{-\rho}=d$, then $t m d \leq 1 / 4$ when $n$ is sufficiently large. By Lemma 2.2, we have

$$
\begin{aligned}
P\left(\left|T_{n}^{*}\right|>\varepsilon\right) & \leq C \exp \left\{-t \varepsilon+C_{0} C_{2} t^{2} \sum_{k=1}^{n} a_{n k}^{2}\right\} \\
& =C \exp \left\{-2 \alpha p \log n+C_{0} C_{2} \frac{(2 \alpha p)^{2} \log ^{2} n}{\varepsilon^{2}} \sum_{k=1}^{n} a_{n k}^{2}\right\},
\end{aligned}
$$

where $C_{0}=E\left(X_{n k}^{\prime}\right)^{2} \leq E X^{2 p}<\infty$. Note that $\log n \sum_{k=1}^{n} a_{n k}^{2}=o(1)$ holds if $\alpha>\frac{1}{2}$. Therefore, by condition (ii), we have

$$
C_{0} C_{2} \frac{(2 \alpha p)^{2} \log n}{\varepsilon^{2}} \sum_{k=1}^{n} a_{n k}^{2} \rightarrow 0, \quad n \rightarrow \infty
$$

Hence, when $n$ is sufficiently large, by (14) and (15), we have

$$
P\left(\left|T_{n}^{*}\right|>\varepsilon\right) \leq C \exp \left(-2 \alpha p \log n+2^{-1} \log n\right)=C n^{-2 \alpha p+1 / 2} .
$$

Then

$$
\sum_{n=1}^{\infty} n^{2 \alpha p-2} P\left(\left|T_{n}^{*}\right|>\varepsilon\right) \leq C \sum_{n=1}^{\infty} n^{-\frac{3}{2}}<\infty
$$

The proof is completed. 


\section{Competing interests}

The authors declare that they have no competing interests.

\section{Authors' contributions}

YFW carried out the proofs of the main results in the manuscript. HD participated in the design of the study and drafted the manuscript. All authors read and approved the final manuscript.

\section{Author details}

${ }^{1}$ College of Mathematics and Computer Science, Tongling University, Tongling, 244000, P.R. China. ${ }^{2}$ School of Mathematical Sciences, Chuzhou University, Chuzhou, 239000, P.R. China.

\section{Acknowledgements}

The authors are grateful to the referees for carefully reading the manuscript and for providing some comments and suggestions, which led to improvements in the paper. The research of Yong-Feng Wu was supported by the Humanities and Social Sciences Foundation for the Youth Scholars of Ministry of Education of China (12YJCZH217) and the Natural Science Foundation of Anhui Province (1308085MA03, 1208085MG121). The research of Hui Ding was supported by the NSF of Education Ministry of Anhui province (KJ2012Z278) and the National Statistics Science Research Project (2012LY153).

\section{Received: 25 March 2013 Accepted: 6 August 2013 Published: 20 August 2013}

\section{References}

1. Hsu, PL, Robbins, H: Complete convergence and the law of large numbers. Proc. Natl. Acad. Sci. USA 33, 25-31 (1947)

2. Blum, JR, Hanson, DL, Koopmans, LH: On the strong law of large numbers for a class of stochastic processes. Z. Wahrscheinlichkeitstheor. Verw. Geb. 2, 1-11 (1963)

3. Dobrushin, RL: The central limit theorem for non-stationary Markov chain. Theory Probab. Appl. 1, $72-88$ (1956)

4. Ibragimov, IA: Some limit theorems for stochastic processes stationary in the strict sense. Dokl. Akad. Nauk SSSR 125, 711-714 (1959)

5. Cogburn, R: Asymptotic Properties of Stationary Sequences. Univ. Calif. Publ. Statist., vol. 3, pp. 99-146 (1960)

6. Sen, PK: A note on weak convergence of empirical processes for sequences of $\varphi$-mixing random variables. Ann. Math. Stat. 42, 2131-2133 (1971)

7. Choi, BD, Sung, SH: Almost sure convergence theorems of weighted sums of random variables. Stoch. Anal. Appl. 5 365-377 (1987)

8. Utev, SA: The central limit theorem for $\varphi$-mixing arrays of random variables. Theory Probab. Appl. 35, 131-139 (1990)

9. Chen, DC: A uniform central limit theorem for nonuniform $\varphi$-mixing random fields. Ann. Probab. 19, 636-649 (1991)

10. Shao, QM: Almost sure invariance principles for mixing sequences of random variables. Stoch. Process. Appl. 48, 319-334 (1993)

11. Rüdiger, K: Strong laws and summability for sequences of $\varphi$-mixing random variables in Banach spaces. Electron. Commun. Probab. 2, 27-41 (1997)

12. Chen, $\mathrm{PY}, \mathrm{Hu}, \mathrm{TC}$, Volodin, $\mathrm{A}$ : Limiting behaviour of moving average processes under $\varphi$-mixing assumption. Stat Probab. Lett. 79, 105-111 (2009)

13. Zhou, XC: Complete moment convergence of moving average processes under $\varphi$-mixing assumptions. Stat. Probab. Lett. 80, 285-292 (2010)

14. Wang, XJ, Hu, SH, Shen, Y, Yang, WZ: Maximal inequality for $\psi$-mixing sequences and its applications. Appl. Math. Lett. 23, 1156-1161 (2010)

15. Wang, $\mathrm{XJ}, \mathrm{Hu}, \mathrm{SH}$, Yang, WZ, Shen, Y: On complete convergence for weighted sums of $\varphi$-mixing random variables J. Inequal. Appl. (2010). doi:10.1155/2010/372390

16. Guo, ML: Complete moment convergence of weighted sums for arrays of rowwise $\varphi$-mixing random variables. Int. J. Math. Math. Sci. 2012, Article ID 730962 (2012)

17. Bradley, RC: On the $\psi$-mixing condition for stationary random sequences. Trans. Am. Math. Soc. 276, 55-66 (1983)

18. Yang, SC: Almost sure convergence of weighted sums of mixing sequences. J. Syst. Sci. Math. Sci. 15(3), 254-265 (1995) (in Chinese)

19. $\mathrm{Wu}$, YF, Zhu, DJ: Complete convergence of weighted sum of $\psi$-mixing random sequences. J. Syst. Sci. Math. Sci. 30, 296-302 (2010) (in Chinese)

20. Yang, YZ, Liu, YY: Strong stability of linear forms of $\psi$-mixing random variables. Chin. J. Appl. Probab. Stat. 27, 337-345 (2011)

21. Hu, TC, Móricz, F, Taylor, RL: Strong laws of large numbers for arrays of rowwise independent random variables. Acta Math. Hung. 54, 153-162 (1989)

22. Taylor, RL, Patterson, RF, Bozorgnia, A: A strong law of large numbers for arrays of rowwise negatively dependent random variables. Stoch. Anal. Appl. 20(3), 643-656 (2002)

23. Baek, Jl, Seo, HY, Lee, GH, Choi, JY: On the strong law of large numbers for weighted sums of arrays of rowwise negatively dependent random variables. J. Korean Math. Soc. 46(4), 827-840 (2009)

doi:10.1186/1029-242X-2013-393

Cite this article as: Wu and Ding: On the complete convergence for arrays of rowwise $\psi$-mixing random variables Journal of Inequalities and Applications 2013 2013:393. 